\title{
Reinforcing high-density polyethylene by phenolic spheres
}

\author{
Lien Zhu' ${ }^{1}$, Di Wu' ${ }^{1}$, Baolong Wang ${ }^{1}$, Jing Zhao ${ }^{1}$, Meihua Liu' ${ }^{1}$,Zheng Jin ${ }^{1, a^{*}}$, Kai Zhao ${ }^{2, b^{*}}$ \\ ${ }^{1}$ Key Laboratory of Chemical Engineering Process \& Technology for High- \\ efficiency Conversion, College of Heilongjiang Province, College of Chemistry Engineering and Materials, Heilongjiang University, Harbin, \\ 150080, PR China. \\ ${ }^{2}$ Laboratory of Microbiology, School of Biological Science and Technology, Heilongjiang University, Harbin, 150080, PR China.
}

\begin{abstract}
Phenolic spheres are synthesized through resorcinol and formaldehyde. The phenolic spheres were blended with HDPE to prepare binary composites. The rheological properties and mechanical properties of the composites were studied. The composite materials have higher tensile strength and impact strength than pure HDPE, which extends the application of the material.
\end{abstract}

\section{Introduction}

High-density polyethylene (HDPE), as a semicrystalline polyolefin, is one of the most common plastics with a wide range of applications such as bottles, pipes and containers [1]. It is a chemically resistant, flexible, easy to process, tough plastic, and most significantly it can be processed at low temperature [2]. However, HDPE cannot approach the typical properties of engineering or high performance polymer due to its low mechanical properties [3,4] which limited its applications in a broader field.

In order to increase the tensile strength of the material, the blending of the polymer with the fiber is an effective way [5]. The principle is that the polymer matrix can transfer stress to the fiber [6]. However, due to the poor interfacial strength between the fiber and the matrix, the tensile strength of the material tends to damage its toughness at the same time.

In this study, phenolic spheres were first synthesized. The prepared phenolic spheres were melt-blended with HDPE to form binary composites, of which rheological properties and mechanical properties of composite materials were analyzed. Due to the well compatibility of phenolic spheres with HDPE, phenolic spheres have successfully acted as reinforcements, resulting in a significant increase in the tensile strength of the composites. Also, due to the proper size of the phenolic spheres, the impact strength of the composite material is improved. These expand the application of materials.

\section{Experiment}

Under room temperature conditions, resorcinol and formaldehyde were mixed in a ratio of 1:2. Anhydrous sodium carbonate was used as a catalyst, and a certain amount of distilled water was added. After stirring, the mixture was added to a three-necked flask and the three-necked flask was placed in an oil bath. In the pot, the set temperature was $85^{\circ} \mathrm{C}$. and the reaction was carried out at a constant temperature for 8 hours. After that, it was washed with anhydrous ethanol, centrifuged, and dried at a constant temperature of $60^{\circ} \mathrm{C}$ for 48 hours to obtain phenolic spheres that were relatively dispersed and uniform in particle size. The experimental reaction device is shown in Fig. 1 and prepared phenolic spherical is shown in Fig. 5(a). The resulting phenolic spheres and HDPE were melt-blended through a twin-screw extruder. After extrusion and granulation, the dumbbell-shaped samples were made using an injection molding machine.

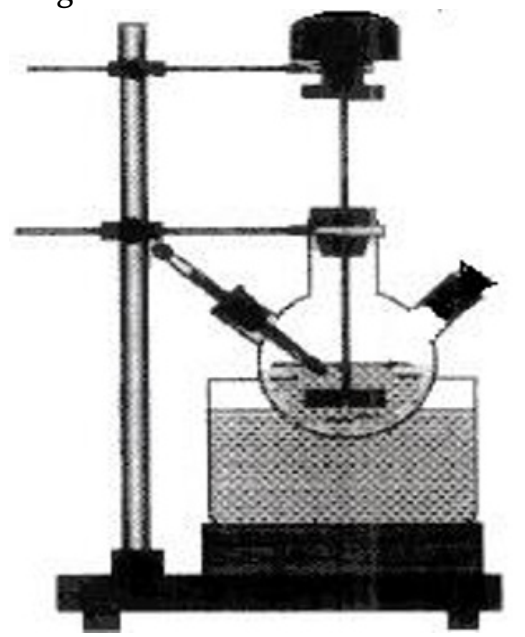

Fig. 1 The reaction device of phenolic spheres

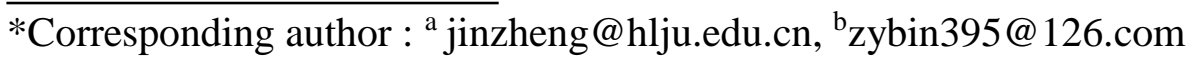


The morphology of all the blends was characterized by scanning electron microscopy (SEM). The samples were fractured after immersing in liquid nitrogen and then covered with gold. Then the fractured samples were observed in a SEM instrument (Model: FEI-INSPECT F, USA) at an acceleration voltage of $5 \mathrm{kV}$.

Tensile propertie of samples was examined according to GB/T 1040.1-06 standard using a tensile testing machine (YHS-229WJ) and the tests were carried out at a displacement rate of $50 \mathrm{~mm} / \mathrm{min}$ at room temperature. The notched izod impact strength of the specimen was determined as per ASTM D256-10 standard using an impact strength testing machine (XJU-2.75J).

Rheological characterization of the samples was carried out at $170{ }^{\circ} \mathrm{C}$ on a rheometer (TA-AR2000ex USA) with a 25 millimeter parallel plate. Strain sweep was carried out to determine the limit on the strain for linear viscoelastic response and the strain range of $0.01^{\sim 100 \%}$ was used. The testing samples with a volume of $20 \times 20 \times 4 \mathrm{~mm} 3$ were prepared by cutting the dumbbell-shaped standard sample. The gap between the two parallel plates in rheology test was set as $2 \mathrm{~mm}$.

The elasticity of samples was measured using a DMS6100 dynamic thermomechanical analyser with alternating stress of the frequency of $1 \mathrm{~Hz}$ at a temperature rise rate of $2^{\circ} \mathrm{C} / \mathrm{min}$. The specimen was prepared by cutting the dumbbell-shaped standard sample with a volume of $35 \times 10 \times 2.5 \mathrm{~mm} 3$.

\section{Results and discussions}

Fig. 2 and Fig. 3 show the dependence of the storage modulus and the loss modulus of the composites with different phenolic sphere content on the frequency. As can be seen, the storage modulus and the loss modulus all show an increasing trend with the increase of the shear rate. The change in storage modulus is due to the greater shear force, the greater the short-term recovery ability of the composite, which showing a significant increase in elasticity. The change in the loss modulus is due to the increased movement of the molecular chain under external forces, which manifests as an increase in system viscosity. In the low frequency region, the storage modulus of the composite material is less than the loss modulus, indicating that the polymer melt is dominated by viscous flow. After the frequency reaches a certain value, the material's storage modulus is greater than the loss modulus, indicating that the polymer melt is dominated by viscous flow.

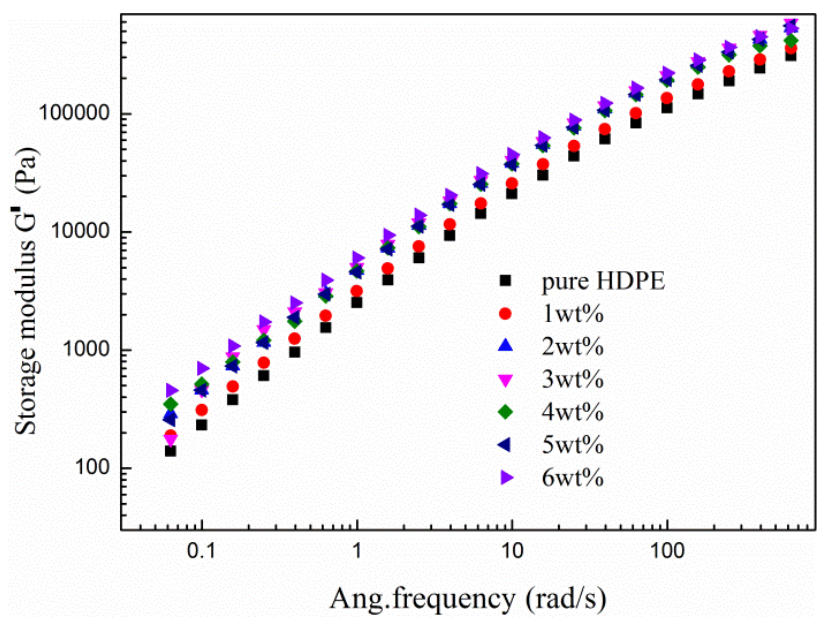

Fig. 2 The plots of storage modulus versus ang.frequency of composites with different content of phenolic spheres

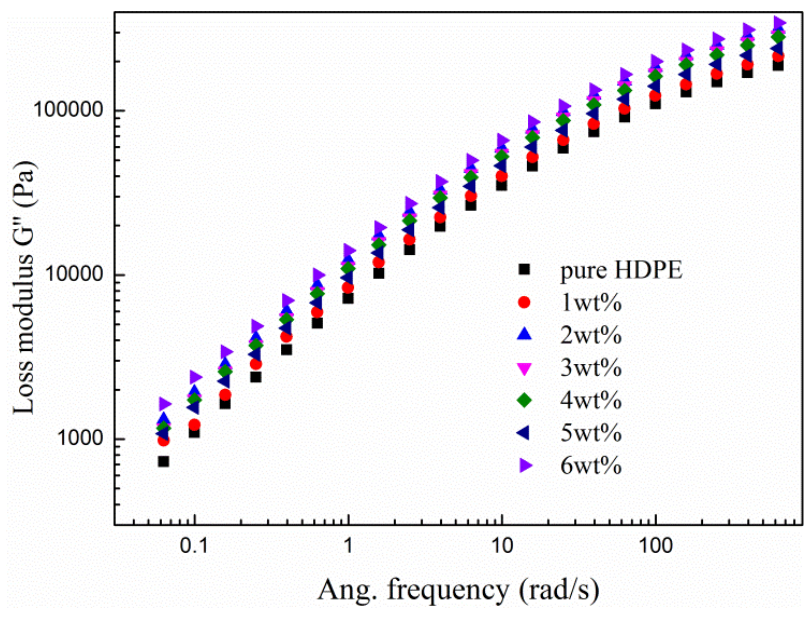

Fig. 3 The plots of loss modulus versus ang.frequency of composites with different content of phenolic spheres

Fig. 4 shows the tensile strength and elongation at break of composites with different phenolic sphere contents. It can be seen from the figure that the tensile strength of the composite increases first and then decreases, reaching a maximum of $25 \mathrm{MPa}$ when the phenolic sphere content is $4 \mathrm{wt} \%$, which is a $17.8 \%$ increase over pure high density polyethylene resin. However, the elongation at break of the composite material decreased significantly with the increase of the phenolic sphere content. When the phenolic sphere content was $4 \mathrm{wt} \%$, the elongation at break decreased by $70 \%$.

There are two main reasons to explain the trend of the tensile strength curve. One is that the heterogeneous nucleation of the phenolic spheres in the composite material increases the crystallinity of the high density polyethylene, which leads to an increase in the tensile strength of the material. The other is related to the degree of dispersion of the phenolic spheres in the composite material, which can be 
visualized by cross-sectional scanning electron microscopy of composites with different phenolic sphere contents in Fig. 5. As can be seen from the figure, when the phenolic sphere content is low, it is independently and homogeneously dispersed in the high-density polyethylene resin matrix, and the particle diameter is about 1 to $1.5 \mu \mathrm{m}$. The phenolic sphere will be pulled out from the resin matrix under the tensile stress, which promotes the transfer of stress and leads to an increase in tensile strength. When the phenolic sphere content exceeds $4 \mathrm{wt} \%$, a part of the phenolic spheres in the composite material stacks to form agglomeration. Since the aggregate is formed by intermolecular forces, a stress concentration will occurs under the tensile stress, causing a decrease in tensile strength. The decrease in elongation at break is due to the fact that the phenolic spheres are organic rigid particles. The addition of the phenolic spheres to the high-density polyethylene resin destroys the flexibility of the matrix molecular chains and causes them to change from flexible to rigid.

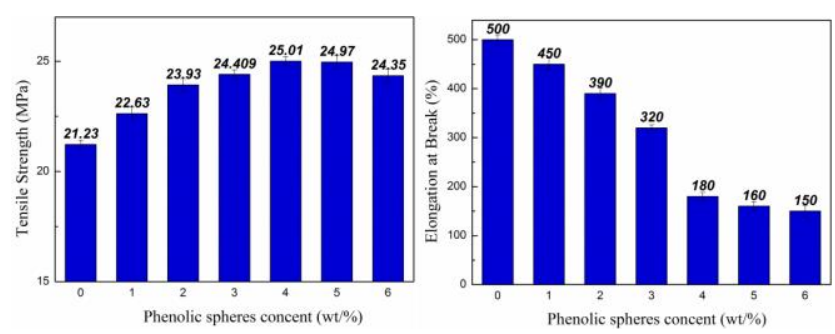

Fig. 4 Tensile strength and elongation at break curves of composites with different content of phenolic spheres

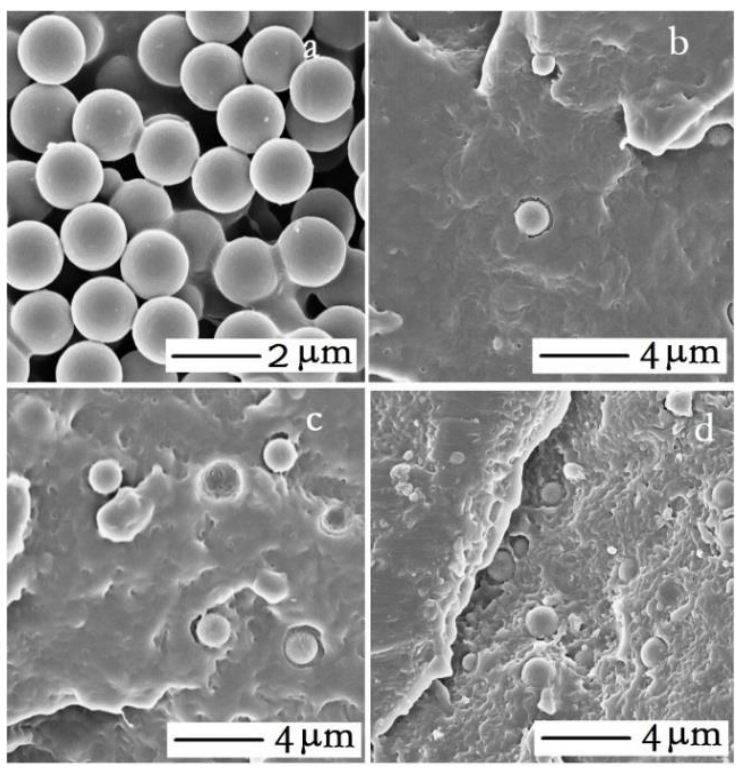

Fig. 5 SEM images of composites with different content of phenolic spheres: (a) phenolic spheres, (b) $2 \mathrm{wt} \%$, (c) $4 \mathrm{wt} \%$, (d) $6 \mathrm{wt} \%$
Fig. 6 shows the impact strength curves of composites with different phenolic sphere contents. It can be seen from the figure that the impact strength increases first and then decreases, reaching a maximum of 32.93 at 4 wt $\%$ which is an increase of $65.98 \%$ compared with pure high-density polyethylene resin. This can be explained by the "silver shear band" theory. When the substrate is subjected to impact stress, the presence of phenolic spheres causes local stress concentration, which induces a large amount of silver lines and shear bands, absorbing a large amount of fracture energy and hindering crack propagation. Also, since the interface strength between the phenolic spheres and the matrix is high, a large amount of fracture energy is absorbed when phenolic spheres are separated from the matrix. All of these lead to an increase in the impact strength of the material. However, when the content of phenolic spheres is too high, more and more silver striations are produced in the composite material. The silver striation is a precursor to submicroscopic fracture of the material. Under the influence of internal and external stresses, the craze gradually develops into a macroscopic fracture, resulting in the decrease of impact strength.

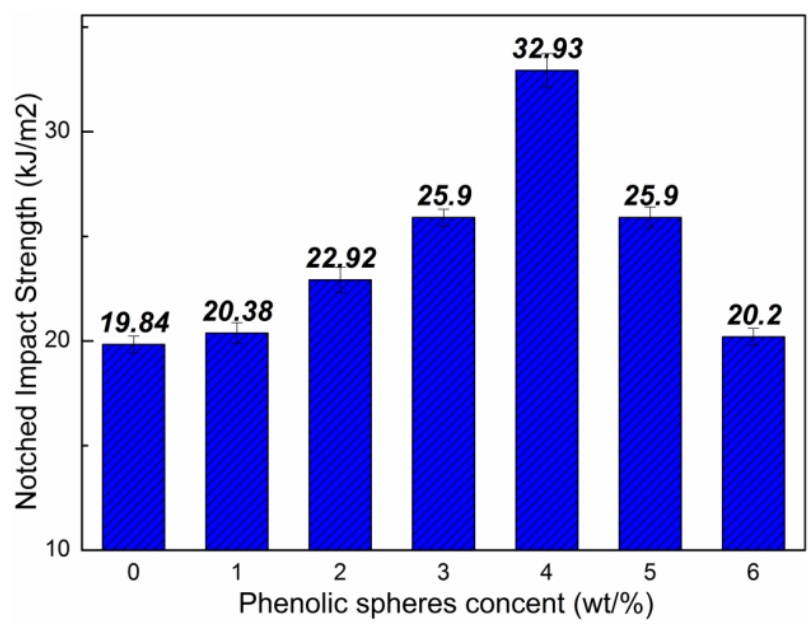

Fig. 6 Notched impact strength of composites with different content of phenolic spheres

Dynamic thermomechanical analysis was used to study molecule movement. In this work, polymer chain movement behavior will be influenced with the addition of fibers. Storage modulus is a parameter characterising molecular chain segment motion. The higher storage modulus, the harder to elastic deform. In other words, storage modulus increasement could enhance resistance capacity to deformation. As displayed in Fig.7, the storage modulus of all composites increases first and then decreases with the increase of phenolic spheres content, reaching the maximum at $4 \mathrm{wt} \%$. This is because when the content of phenolic spheres is small, the microspheres form a 
good dispersion effect in the matrix, and the independent microspheres can effectively prevent the movement of the molecular chains. The deformation of the segments can not keep up with the change of stress, so that the deformation elastic energy storage is gradually generated. When the content of phenolic aldehydes exceeds $4 \%$, the microspheres begin to aggregate together by intermolecular forces. The chain motion makes it easier for the deformation to keep up with the change in stress and the storage modulus is reduced. This phenomenon signified that the resistance capacity to deformation of composites enhanced with the addition of phenolic spheres.

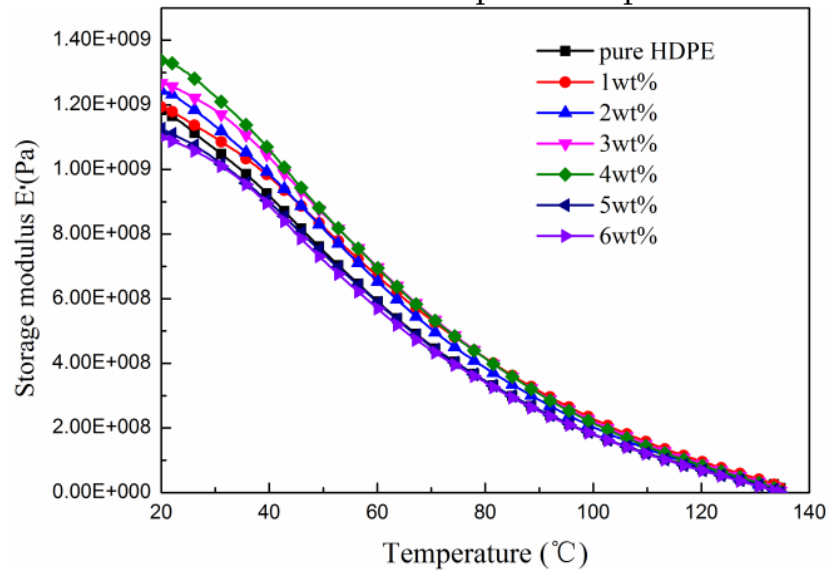

Fig.7 Storage modulus of composites with different content of phenolic spheres

\section{Conclusion}

With the addition of the phenolic spheres, the storage modulus, loss modulus, tensile strength and impact strength of the materials have all improved significantly, which extends the application of the material.The best mechanical properties are obtained when the phenolic sphere content is $4 \%$.

\section{Acknowledgements}

The authors gratefully acknowledge the National Key Research and Development Program of China (Grant Numbers 2017YFD0500706 and 2017YFD0500603), National Natural Science Foundation of China (Grant Numbers 31570929 and 31771000), Natural Science Foundation of Heilongjiang Province (Grant Number C2017058), Innovation Foundation of Harbin (Grant Number 2017RAXXJ001), Graduate Student Innovation Research Project Funding of Heilongjiang University (Grant Number YJSCX2018061HLJU), Students Innovation and Entrepreneurship Training Project of China (Grant Number 201810212020 ) and Key Scientific Technological Planning Project of Harbin (Grant Number 2016AB3BN036).
1. J. Zhang, G. M. Rizvi, C. B. Park. J. Mater. Scimater. 46,11(2011)

2. K. Sewda, S. N. Maiti. Polym. Bull. 70,10(2013)

3. A. V. Ortiza, J. G. Teixeiraa, M. G. Gomesa, R. R. Oliveiraa, F.R.V. Díazb, E.A.B.Moura, Appl. Surf. Sci. 310,8(2014)

4. C. L. Simões, LMC Pinto , C.A Bernardo, C. L. Pinto, L. M. Bernardo, C. A. Waste. Manage. Res.32,5(2014)

5. L. Xia, T. Zhang, Z. Chai, X. Hu, F.Jin, G. Wen. J. Eur. Ceram. Soc.36,14(2016)

6. L. Zhu, D. Wu, B. Wang, J. Zhao, Z. Jin. Pigm. Resin. Technol. 47, 1(2017)

\section{References}

